\title{
Compatibilization in Two-Component Injection Molding by Means of Split Reactions with Varying Reactive Sites - a Monte-Carlo Simulation
}

\author{
Andreas John*, Jürgen Nagel, Gert Heinrich
}

Leibniz Institut für Polymerforschung e. V., Hohe Strasse 6, 01069 Dresden, Germany

\begin{abstract}
Adhesion of immiscible polymers during two-component injection molding may be improved by transreactions of properly functionalized molecules in situ using the thermal energy of the melts. These reactions must provide a sufficient conversion of reactive monomers during the short cooling time down to the glass temperature and within the small spatial region of the interface width to create as much as possible interconnecting chemical links between the components on the molecular level. To investigate these processes, we performed Monte-Carlo (MC) simulations based on the three dimensional coarse-grained Bond Fluctuation Model (BFM) in a two-phase system. We studied split type reactions exhibiting reactive monomers at different sites (End, Middle, Random) of the polymers governed by activation energies of $\mathrm{E}_{\mathrm{A}}=$ $0,1,3,5$ and $7 k_{B} T$. For the reacting systems several physical properties like consumption, radius of gyration, concentration profiles or the distribution of the degree of polymerization were calculated as a function of time. Additionally, different functions for the description of adhesion on the molecular level were adopted and calculated depending on reaction type, activation energy and degree of consumption. From the results those chemical reaction types were deduced, which should be most suitable for the compatibilization in two-component injection molding.
\end{abstract}

Keywords: Injection molding, reactive compatibilization, monte-carlo simulation.

\section{INTRODUCTION}

In the polymer manufacturing industries blending is a widely used technique to combine different polymers in one product. In this way it is usually possible to improve the performance of the composite and to take advantage of the most favourable properties (e.g., thermal stability, elasticity, permeability) of both the materials [1].

Unfortunately, the thermodynamical miscibility of most polymer melts is very low, and the strength of the interface between the two materials is widely determined by Van der Waals forces between the polymer molecules. Additionally, chains being located within the interpenetration depth will increase their entropy by crossing the interface and so contribute to the stability of the two-phase system. Physical entanglements improving the adhesion can only be performed in the short cooling time down to the glass temperature of few seconds within the nanometer [2] region of interdiffusion and thus they are little pronounced.

But in many cases physical adhesion alone does not lead to the performance properties for everyday use. However, an improvement of adhesion of different polymers may be expected generally by (i) flow induced acceleration of the reactive coupling rate in melt mixers [3], (ii) deposition of diblock copolymers of both the components inside the interdiffusion boundary layer [4-6] or (iii) the in situ formation of chemical bonds between the two components during a reactive extrusion process [7-9].

\footnotetext{
*Address correspondence to this author at the Leibniz Institut für Polymerforschung e. V., Hohe Strasse 6, 01069 Dresden, Germany; Tel: +49 351 4658 751; Fax: +49 3514658 752; E-mail: john@ipfdd.de
}

This latter favourable technique also may be successfully extended to the injection molding procedure and in this way take advantage of the thermal energy of the melts [10].

In the short cooling time and the small dimension of the boundary layer the process must lead to a sufficient amount of copolymers by interdiffusion and transreaction. Only those chemical reaction types satisfying these requirements will become technically relevant.

Among the reaction types that were intensively studied in the past are the coupling reactions of monoendfunctionalized polymers $\mathrm{A}$ and $\mathrm{B}$ forming simple copolymers $\mathrm{AB}$ (Link). Because of the low concentration of the reactive groups these reactions will lead to only relatively small reaction rates at the interface between the polymers and the variety of reaction products is restricted to diblock copolymers [11-13].

On the other hand, polycondensates may have many functional groups within the polymer chain and, therefore, give rise to more complicated reaction types like splitting (Split) or branching reactions (Branch), which also may be combined. Due to the higher concentration of functional groups compared to the simple Link reaction, these latter reaction types under favourable conditions may yield greater reaction rates and therefore could become more important for building in situ reaction products in the two-component injection molding process [14].

Because of the small time and space dimensions being available for these reactions, experimental investigations are very difficult to perform and so the theoretically analytical approach and also simulation techniques seem to be a useful method to get some deeper insight into the fundamental statistical processes leading to bond formation in the interface region. 
However, due to the simplicity of the reaction mechanism the reaction type Link considering only low concentrations of reactive monomers was subject to analytical [15-18] and simulation [19-20] studies several times, whereas the other types Split and Branch were investigated only less and more qualitatively [21] by means of simulations so far. On the other hand, the technical processes, mentioned above, require high growth rates of the reaction products and therefore must be performed at high concentrations of reactive chains.

Because of their simplicity maintaining the basic features of polymeric melts many simulations were performed by means of Monte-Carlo techniques using the Bond Fluctuation Model (BFM) [22-25]. This coarse-grained three dimensional lattice model retains the important features of a polymeric system like (i) connectivity of the monomers, (ii) excluded volume interaction, (iii) a short range thermal interaction potential, and allows to simulate essential properties of dense polymeric melts including the crossover behaviour from Rouse to reptation dynamics [29]. We qualitatively investigated in a previous study [21] fast (activation energy $\left.E_{A}=0 \begin{array}{lll}0 & k_{B} T\end{array}\right)$ interface reactions between immiscible reactive polymers including complex reaction mechanisms like interchain and intrachain transreactions. The formation kinetics of those products (including ring copolymers) because of auxiliary reactions is no unique function of the consumption of reactive monomers $\mathrm{C}$ forming the ends of the $\mathrm{B}$ polymers. But this is necessary in the attempt to develop a quantitatively phenomenological kinetic model. Although these models in the first stage are as possibly as simple, they can describe technically relevant reaction types.

Therefore, in a later work [26] we quantitatively investigated the interface reaction behaviour $\left(\mathrm{E}_{\mathrm{A}}=0,1,3\right.$ and 5 $\mathrm{k}_{\mathrm{B}} \mathrm{T}$ ) between two immiscible polymers A and B exhibiting the reaction types Link, Split and Branch under technically relevant concentrations of reactive chains. It was shown, that the very first stage of the reaction in a good approximation can be described by a bimolecular time rule. Several products of the reaction Split are able to penetrate the volume B phase and are expected to extraordinarily contribute to the adhesion performance of the composite in the twocomponent injection molding.

Recently [27], we studied the reactive compatibilization (blending) of randomly functionalized backbone polymers with end-functionalized graft polymers forming cocontinuous nanostructured microphases. We have compared reacted and non-reacted systems under the same conditions. For the non-reacted system at low temperatures phase separation was observed. In the system with grafting reactions macroscopic phase separation was inhibited even if the consumption of reactive sites was only $50 \%$. The calculated structure factor indicated a distinct difference between the two simulation states in accordance with the 3D visualization of the system and the box-counting method.

However, in all the cases the relation between the chemical reaction types on the molecular level and the consequences concerning the macroscopic strength behaviour is not well understood.
There are several concepts relating structure to strength resting on the assumption, that the greater the amount of mixing or chain interpenetration at the interface is, the greater will be the strength, developed by this structure [2]. Therefore, bridge concepts counting (i) the number of intersecting chains, (ii) the number of bridges crossing the interface, (iii) the average monomer interpenetration depth, or (iv) the number of monomers crossing the interface seem to be useful to develop functions describing macroscopically measurable properties on the molecular level.

Therefore, in our present investigation as extension of our previous work [26] we compare the relative strength of composites created without reaction, and as result of three different interface reaction types between functionalized immiscible polymer melts, respectively. We have adopted several bridge concepts describing the degree of interpenetration on the molecular level to obtain a measure of strength on the macroscopic scale. The aim of our work is to estimate the usefulness of the chemical reaction types to create composites of high strength during the two-component injection molding. We have consciously decided to make all the monomers of one chain type (A) reactive to make the possible effects of strength enhancement for the adoption of polycondensates most clearly pronounced.

\section{MATERIALS AND METHODOLOGY}

Within the framework of the BFM [22-25] each segment of a polymer allocates the eight corners of a unit cell in a simple cubic lattice with lattice unit $a_{0}$ and no site can be doubly occupied. All the length data given in the text will be in units of $a_{0}$. The centres of the cubes are connected by bond vectors. Serving the condition of the excluded volume and preventing the bond vectors from crossing during the random motion of the monomers leads to a set of 108 bond vectors with length squares of 4, 5, 6, 9 and 10 including 87 bond angles altogether. The attempted move of a monomer unit will only be accepted, if (i) the excluded volume is retained, (ii) the new bonds are allowed in the BFM and (iii) the change in energy satisfies the Metropolis criterion [28]. We have decided to create a model of the immiscible $A / B$ system consisting of 876 chains of both types A, B with degrees of polymerisation of $P=32$. All the 56064 monomers are located in a box of size $L_{X} \cdot L_{Y} \cdot L_{Z}=(-120:+120)$. $(-30:+30) \cdot(-30:+30)$ representing a volume fraction of nearly 0.5 , for which the BFM reproduces many characteristic properties of a dense polymeric melt. Periodic boundary conditions were imposed on all three spatial directions to simulate a system of infinite size. In this way, a sufficiently large diffusion path in $x$ direction is provided to ensure the unperturbed volume conditions for comparison intentions. Additionally, the periodic boundary conditions in $x$ direction create $1+2 \cdot \frac{1}{2}=2$ interfaces, which act as a doubled reaction surface. The initial configuration was equilibrated without the energy criterion using $10^{6}$ Monte-Carlo steps (MCS). Then, the chains were separated into type A and type B depending on whether the $x$-coordinate of the centre of mass of the chain was less (A) or greater (B) zero, so that the mathe- 
matical interface between the A/B phases is the yz-plane at $x$ $=0$.

The short range pair interaction potential will be usually calculated between the monomer units A, B and the surrounding 54 segments in a distance of $r \leq \sqrt{6}$ by an energy term $\varepsilon=\varepsilon_{A B}=-\varepsilon_{A A}=-\varepsilon_{B B}$. So the monomers of the same type attract and monomers of different type reject each other. The degree of polymerisation $P=32$ is well below the value, where the first hints for reptation like motion are observed. We have specified the energy to be $\varepsilon=0.1 k_{B} T$ and so the incompatibility corresponds to $\chi P \approx 17$, which is well inside the strong segregation limit of a binary polymeric blend. The configuration again was equilibrated using $10^{6}$ MCS until the static properties bond length $l$, end-to-end distance $R_{E}$, radius of gyration $R_{G}$ became constant and the equilibrium interface structure was established. Continuing the equilibration, nearly all $10^{4}$ MCS statistically independent configurations were extracted as start configurations for the simulation runs including the chemical reaction.

The chemical reactions being under consideration in this study are non-reversibly and are governed by activation energies of $\mathrm{E}_{\mathrm{A}}=0,1,3,5$ and $7 k_{B} T$, which are implemented in terms of an additional Metropolis criterion. All monomers in chains of type $\mathrm{A}$ are reactive and are denoted as D. The D chains are randomly split up forming usually a unsymmetrical diblock copolymer and a fragment of $\mathrm{D}$. As result of subsequent reactions, also triblock copolymers or single monomers can arise. All chains of type B do not split and we have called the reaction types Split-End, Split-Mid and Split-Ran depending on the position of the only reactive monomer $\mathrm{C}$ in the chain - at one end (End), in the middle (Mid), and randomly chosen (Ran). Note, that in this way the consumption of a reactive monomer $\mathrm{C}$ is a unique measure for the progress of the reaction and leads to the formation of exactly one copolymer. Each time a reaction event occurred, the exact MCS was recorded in a log file to construct the nonlocalized consumption of reactive monomers $\mathrm{C}$ as function of time.

In Fig. (1) the three reaction types Split-End, Split-Mid and Split-Ran are presented schematically.

The reaction Split-End directly corresponds to the reaction type Split in our previous work [26] to make the observations for the new reaction schemes Split-Mid and Split-Ran directly comparable to former obtained results.

After a pre-reaction simulation run of about $10^{6} \mathrm{MCS}$ the chemical reactions were initiated at $t=0$ (similarly, as we would use a radiation flash in the experiment to activate the reaction centres) and were processed more than $2 \cdot 10^{5}$ MCS (201813) just exceeding the Rouse time $\tau_{R}$ of chains with degree of polymerisation $P=32$. The time $\tau_{R}$ was estimated from a simulation run without reaction to be $\tau_{R} \approx 10^{5}$ MCS. For each of the twenty tasks (no reaction, reaction types Split-End, Split-Mid and Split-Ran with activation energy of $0,1,3,5$ and $7 k_{B} T$, respectively) 100 simulation runs were processed to ensure sufficiently statistical certainty. The simulations were performed on eight standard personal computers (Pentium IV, 2.8 GHz, 512 MB RAM) consuming 6 hours CPU time per simulation run ( $\approx 1.5 \cdot 10^{6}$ attempted moves per second). The $170 \mathrm{MB}$ data for the configurations at several MCS were stored on disk for further evaluation. The simulation program itself was written in DELPHI, for the extraction of several physical properties of the system like end-to-end distance, radius of gyration, displacements of single and multiple bound monomers and

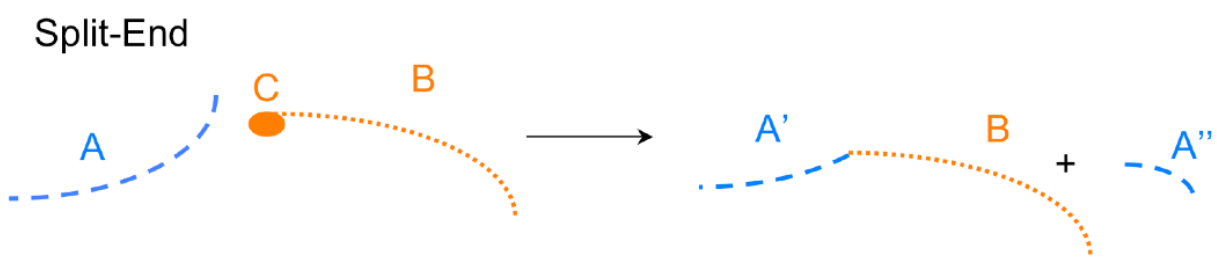

\section{Split-Mid}
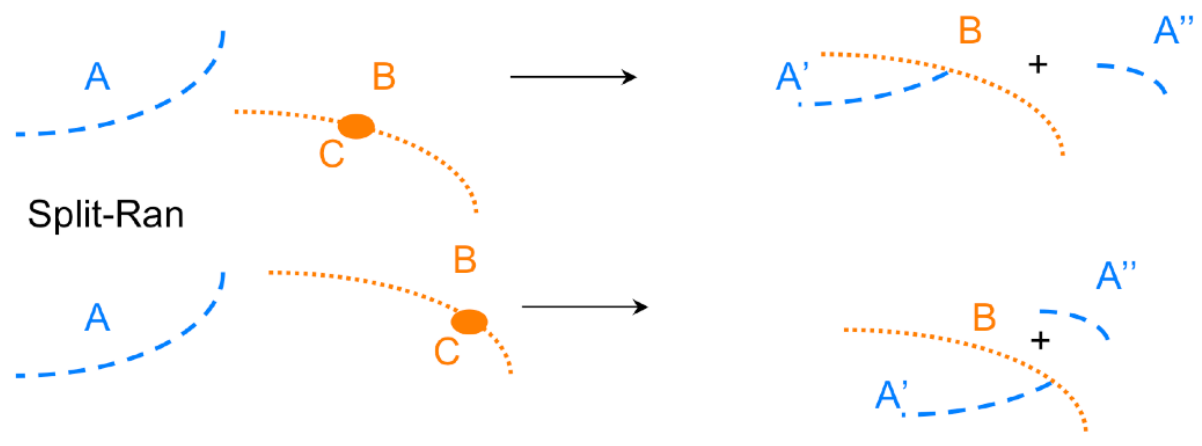

- - reactive monomers

Fig. (1). Schematic representation of the reaction types Split-End, Split-Mid and Split-Ran. 
the reaction events as a function of time a Compaq Fortran90 program was written and used to process the configurations of one of the twenty tasks at once.

\section{RESULTS AND DISCUSSION}

First, after equilibration of the two-phase system, we investigated three selected static properties of the demixed blend in an independent simulation run without reactions. The end-toend distance $\left\langle R_{E}^{2}\right\rangle^{1 / 2}=16.8 \pm 0.8$, the radius of gyration $\left\langle R_{G}^{2}\right\rangle^{1 / 2}=6.9 \pm 0.3$ and the bond length $\left\langle\mathbf{l}^{2}\right\rangle^{1 / 2}=2.62 \pm 0.01$ are close to values from our previous work [26] $\left(\left\langle R_{E}^{2}\right\rangle^{1 / 2}=\right.$
16.4, $\left.\left\langle R_{G}^{2}\right\rangle^{1 / 2}=6.8,\left\langle\mathbf{l}^{2}\right\rangle^{1 / 2}=2.62\right)$ and are somewhat less than those found for a homogeneous system of the same degree of polymerisation and volume fractions of the monomers without interaction of 17.1, 7.0 and 2.64 [29], respectively. But, considering the remaining statistical uncertainty, we believe these differences to be not significantly.

\section{REACTION CONVERSION}

In Fig. (2) we present a survey of all the consumption curves of the reactive $\mathrm{C}$ monomers as a function of time in a doubly logarithmic scale.

The greater the activation energy was specified, the more the onset of the reaction (with consumption $=1$ ) is shifted to

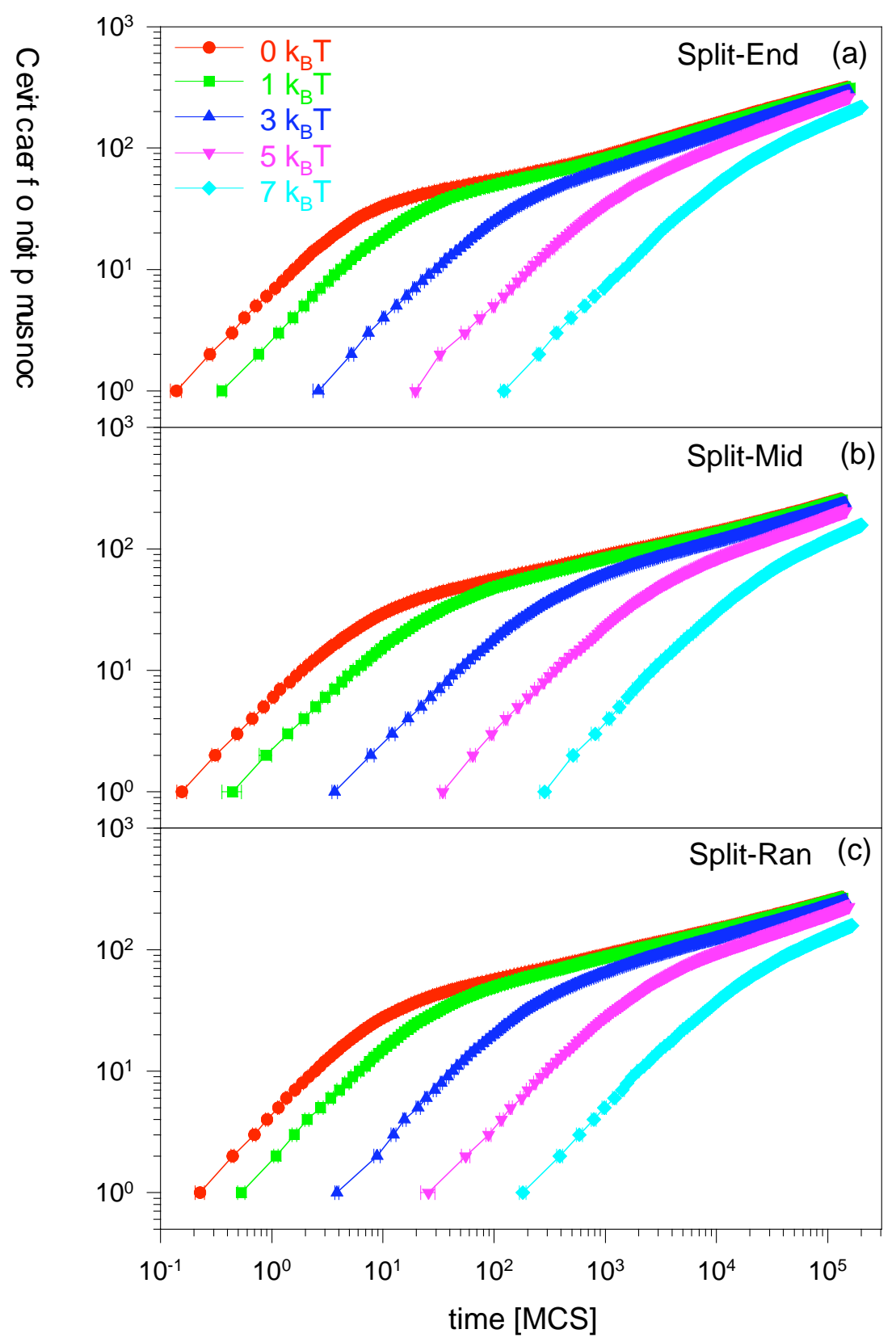

Fig. (2). Consumption of reactive C monomers for the reactions Split-End (a), Split-Mid (b) and Split-Ran (c) at activation energies of 0, 1, 3, 5 and $7 k_{B} T$, respectively. 
larger times. Applying the activation energy $E_{A}=0 k_{B} T$ already after a very small time monomer contacts leading to chemical reactions are observed. This is the very important time section for the technical process of two-component injection molding, since the melt will cool down very rapidly and only in the low-viscous state chain movements and reaction events are possible. Therefore, for our simulation this time stage is of special interest, since the diffusioncontrolled section will probably not be of importance within the cooling time. We find a typical appearance of the curves forming a first part at lower times with a slope of $\approx 0.8-1.0$ and a second part at later times with a slope of $\approx 0.2-0.3$ for all the reaction types. Similar to formerly discussed results [26] we attribute the first part of the curves (that we call kinetic controlled) to the consumption of reactive $\mathrm{C}$ monomers by local and frequent monomer contacts due to conformation alterations around the reaction zone at $x \approx \pm R_{G}$. Neglecting transport phenomena, this behaviour may be described by a simple bimolecular time rule $\frac{d n_{C}}{d t}=-k \cdot n_{C} \cdot n_{D}$, where $n_{C}$ and $n_{D}$ are the total numbers of reactive monomers $\mathrm{C}$ and $\mathrm{D}$ being available in the reaction zone, respectively, and $k$ is the reaction rate constant (in $\mathrm{MCS}^{-1}$, because $n_{C}, n_{D}$ are dimensionless in this context). Clearly, this simple ansatz will not describe the crossover behaviour to the second part in time, but it provides the possibility to calculate the intrinsic kinetic rate constant of the process in the very first stage of the reaction. The second part (that we call diffusioncontrolled) we believe to be the result of rate dominating transport processes of reactive monomers from the volume phases into the reaction zone by Fick's diffusion. In our opinion the slope of $\approx 0.2-0.3$ can not be explained in a simple straightforward manner based on the known displacement behaviour of the monomers. But it can be derived from the previously introduced reaction-diffusion model [26] $\frac{1}{a_{0}} \frac{\partial c_{C}}{\partial t}=D(t) \frac{\partial^{2} c_{C}}{\partial x^{2}}-k c_{C} c_{D}$, when the calculated monomer concentration profiles $c_{C}(x, t)$ for proper values of $D$ and $k$ are integrated. To obtain the predicted consumption curves for reactive $\mathrm{C}$ monomers for each configuration (time step) we calculate the total number $n_{C}(t)=\int_{-120}^{+120} c_{C}(x, t) d x$ of nonreacted $\mathrm{C}$ monomers and create the difference to the value at the origin in time $u_{C}(t)=n_{C}^{0}-n_{C}(t)$ - the consumption. As we found numerically, the curves $u_{C}(t)$ exhibit for small times in the doubly logarithmic scale a slope of $<1$, but in later times below the Rouse time a slope of $\approx 0.2-0.3$, as to be observed in the simulations. Finally, we have calculated the reaction rate constants depending on the activation energy according to the procedure described in our previous work [26]. The results are shown in Fig. (3) in conjunction with the order of the statistical uncertainties, which we have estimated from the standard deviations of the reaction time determination by error propagation.

The curves in a good approximation yield straight lines with a slope of $\approx-1$ for $\ln (k)$ (for convenience, we have shown the lines as $\log (k))$ in the case of the reactions SplitEnd and Split-Ran following a simple Arrhenius ansatz $k=k_{0} \cdot \exp \left(-E_{A} / k_{B} T\right), k_{0}$ is the pre-exponential factor. The rate constants for the reaction Split-Ran are systematically lower than the constants for the reaction Split-End. This result arises, since the end-monomers, being responsible for the rate of the latter reaction, are somewhat more mobile than the twofoldly bound reactive monomers $\mathrm{C}$ in the reaction scheme Split-Ran. Contrary to this behaviour, the line for the reaction Split-Mid obviously exhibits a steeper slope

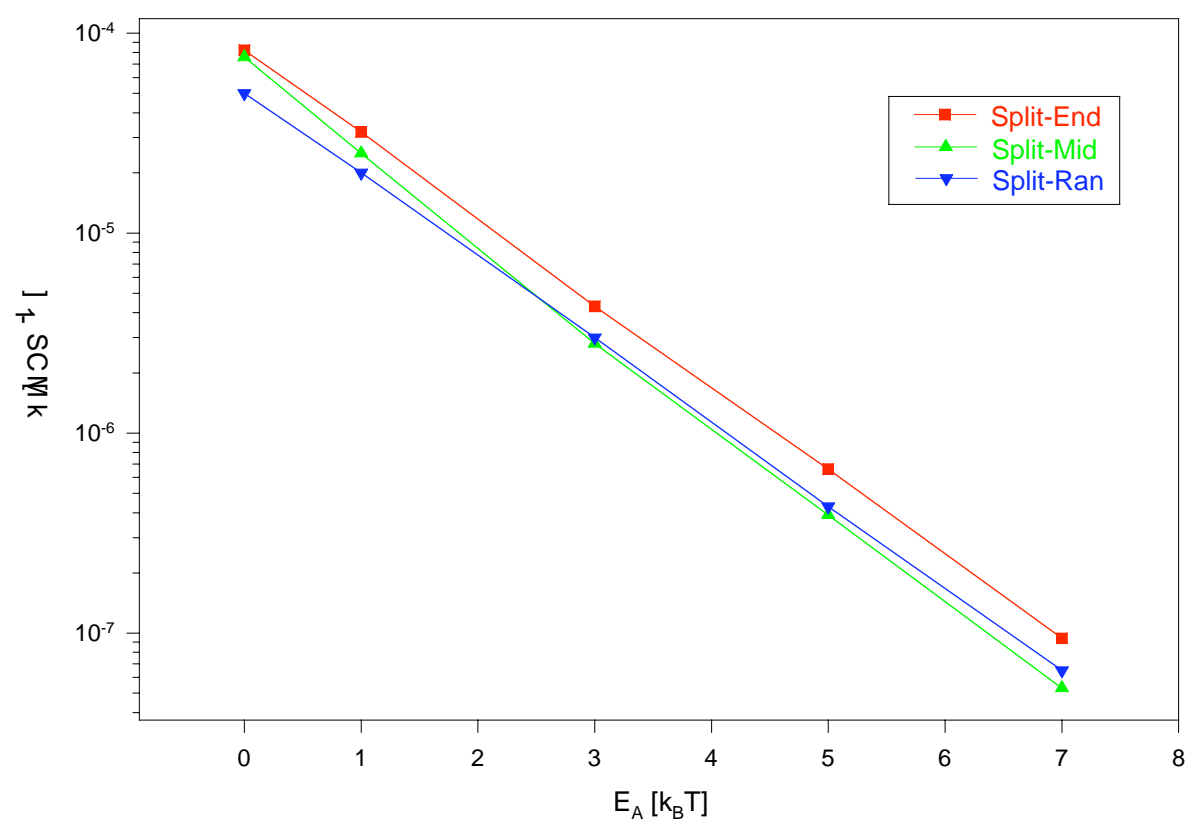

Fig. (3). Rate constants $k$ (in $\mathrm{MCS}^{-1}$, see text) from the model of reaction zone [26] for the reactions Split-End, Split-Mid and Split-Ran as function of the activation energy. 
indicating a more sensitive dependence on the activation energy than the reactions Split-End and Split-Ran. The reason for this behaviour can be found in the restricted mobility of the central reactive $\mathrm{C}$ monomer, which also may be shielded by surrounding monomers of the same chain. However, for a low activation energy and in the very first stage of the consumption the reaction Split-Mid has a higher reaction rate than the reaction Split-Ran. This effect may probably be attributed to the enrichment of chain ends in the interface region in conjunction with the corresponding formation of a zone of loops containing centricly located reactive $\mathrm{C}$ monomers somewhat deeper in the interpenetration depth of the B polymers. Note, that the rate constants $k$ are determined for the very early state of the reactions, where the influence of reaction products is small. Later, due to the complexity of the structure and distribution of di- and triblock copolymers, the rate is dependent both on $E_{A}, t$ and the distribution of reaction products, and so is no longer predictable in a straightforward manner. Moreover, a detailed analysis of the consumption curves of the three reaction types for a specific activation energy shows, that the curves repeatedly change their sequence. This means an exchange of the relative speed of the reactions as function of time and so the dependence on the structure and distribution of the corresponding reaction products. Therefore, we believe the simulation to be the only way to obtain an (at least model) picture of the processes being of importance for the two-component injection molding technique.

For further discussions, we will now focus on results, which we have obtained for the activation energy $E_{A}=0 k_{B} T$, since all the effects of the chemical reaction will be most pronounced in this case of the largest rate constant of the reaction type, being under consideration. For higher activation energies we have obtained no further qualitative changes, but only alterations due to the lower reaction rate resulting in a deceleration of all the effects in time.

\section{DISTRIBUTION OF THE DEGREE OF POLYMERIZATION}

In Fig. (4) we present the distribution of the degree of polymerization (the molar fraction $\varphi(P)$ of molecules with degree of polymerization $\mathrm{P}$ as function of $\mathrm{P}$ ) for the three reaction types after termination of the simulation.

The distributions of the degree of polymerization are very similar, and we find gaps at $\mathrm{P}=31$ and $\mathrm{P}=65$, as well as maxima at $P=32,64$ and 66 . Free monomers $P=1$ can arise from the attack of a $\mathrm{B}$ chain with reactive monomer $\mathrm{C}$ on the second last monomer of a $\mathrm{A}$ chain and the molecules $1<\mathrm{P}<31$ are other fragments of broken A chains. The gap at $\mathrm{P}=31$ is caused by our (arbitrary) agreement in the simulation program to let end-to-end contacts of $\mathrm{C}$ and $\mathrm{D}$ monomers lead to a simple addition forming symmetric diblock copolymers $\mathrm{AB}$. The maximum at $\mathrm{P}=32$ is due to non reacted $\mathrm{A}$ and $\mathrm{B}$ chains, whereas the molecules at $\mathrm{P}=33$ are products arising from the recombination of free A monomers with non reacted $B$ chains mainly within the near-interface region of the volume phase $\mathrm{B}$, where the free monomers $\mathrm{A}$ may penetrate few times after their release. Since B chains cannot be split, the products $34 \leq P \leq 64$ are B chains connected with fragments of A up to symmetric diblock copolymers at $\mathrm{P}=64$. The latter are formed frequently due to the enlarged mobility of the end-monomers. The gap at $\mathrm{P}=$ 65 is caused by the same reason as discussed above for the gap at $\mathrm{P}=31$, since a splitting of A chains at the last monomer of the chain or the fragment is not permitted by the simulation program. Finally, the molecules at $66 \leq P \leq 96$

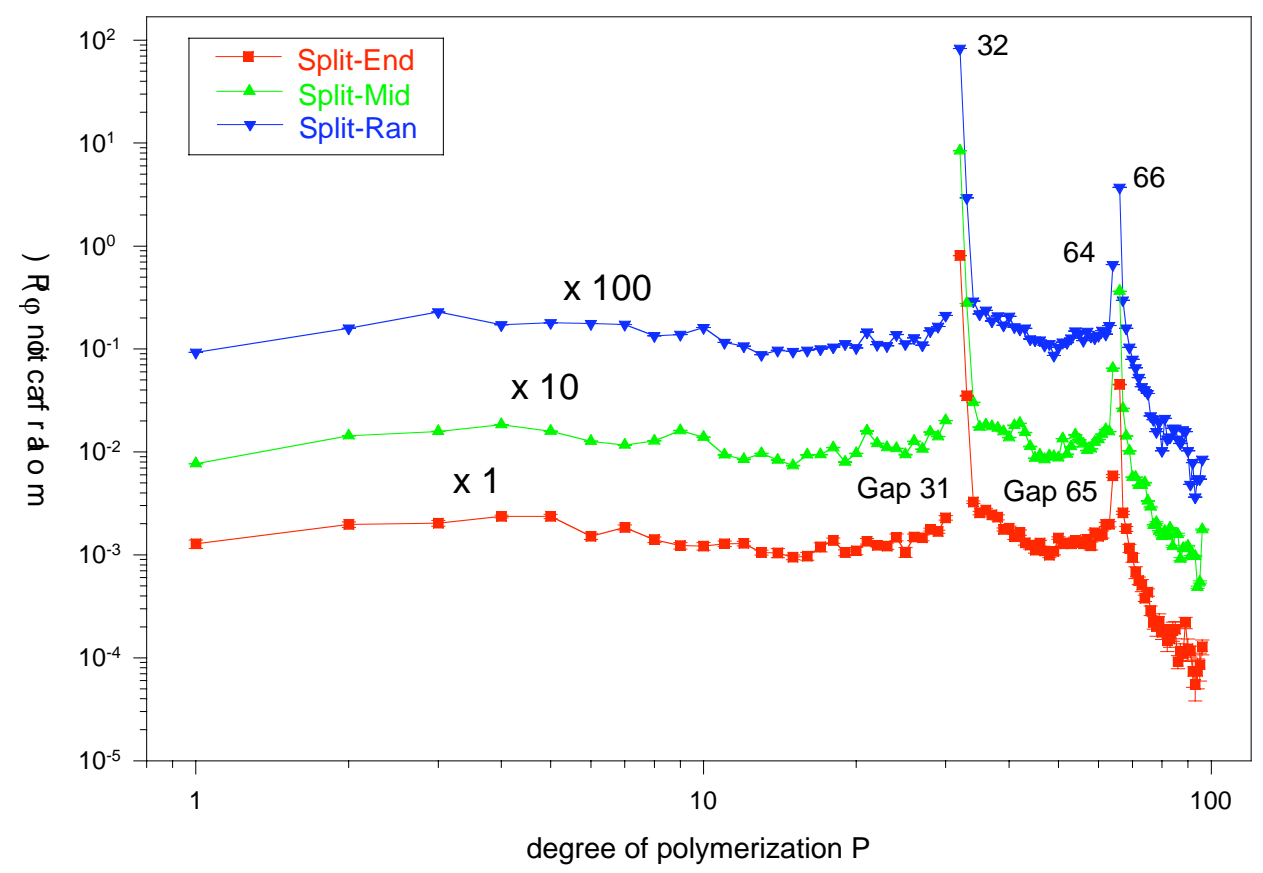

Fig. (4). Distribution of the degree of polymerization for the reactions Split-End, Split-Mid and Split Ran at activation energy $E_{A}=0 \quad k_{B} T$ after termination of the simulation at time $=201813$ MCS. (The multiplication factors are for convenience only). 
are triblock copolymers incorporating two $\mathrm{B}$ chains and variably long A fragments up to complete, symmetric triblock copolymers with $\mathrm{P}=96$. This is also the upper limit of the degree of polymerization for the three reaction types. The reason for the relative maximum at $\mathrm{P}=66$ is the result of the fact, that a triblock copolymer containing two innermost A monomers cannot be fragmented anymore and so is enriched as a final product at later time of the simulation, as it is the case for our termination time $\mathrm{t}=201813$ MCS. From the distribution functions $\varphi(P)$ for each configuration we have calculated the mean degree of polymerization $\langle P\rangle=\sum_{P=1}^{96} P \cdot \varphi(P)$ for the systems as a function of time. Due to the formation of larger molecules and also because the total number of objects decreases, we obtained a growth of the mean degree of polymerization from $\langle P\rangle=32$ at $t=0$ to about $\langle P\rangle \approx 34$ at termination time for the three reaction types. We have also calculated the local distribution $\langle P(x)\rangle$ at the termination time, where all three reaction types exhibit a maximal degree of polymerization of $\langle P(x)\rangle \approx 50$ at approximately $x=10$, a more precise inspection of the curves gives indications for the existence of two maxima separated by $\Delta x \approx 5$. Since the products of the reaction types Split-Mid and Split-Ran contain at least one threefoldly bound monomer having a lower mobility than twofoldly bound monomers, the front of the distribution curve for the reaction SplitEnd is shifted more into the B phase than those of the other two reaction types. We will discuss the reason for the twomaxima structure later, when we inspect the concentration profiles for non-reacted and reacted $\mathrm{C}$ monomers. These latter monomers label the distribution of the reaction products within the simulation box. The maxima of the curves $\langle P(x)\rangle$ are located to the right of the mathematical interface within the B phase. Obviously, because the reaction products always contain intact $\mathrm{B}$ chains, but only fragments of $\mathrm{A}$ chains. These unsymmetrical diblock and triblock copolymers become now more or less miscible with the B polymers. On the other hand, to the left of the mathematical interface we find a minimum of the degree of polymerization due to the accumulation of pure A fragments with $\mathrm{P}<32$. Especially remarkable to the authors is the behaviour of the curves extending into the volume phases $\mathrm{A}$ and $\mathrm{B}$, respectively. As expected, to the right within the B phase we find the convergency of the curves approaching $\langle P(x)\rangle=32$, but to the left a lower value of approximately $\langle P(x)\rangle \approx 31-32$ is obtained. This lower value is the result of the distribution of free A fragments with degree of polymerisation of $P \leq 5$ within the A volume phase, where they can penetrate because of their high mobility. Note also, that we look at the results after terminating the simulation at $\mathrm{t}=201813$ MCS. If we inspect the curves shortly after starting the reaction, there is no indication for a lower degree of polymerization to the left of the interface within the A phase.

In Fig. (5) we show the concentration profiles for the non-reacted (a) and the reacted (b) $\mathrm{C}$ monomers after termination of the simulation, respectively.
Whereas the reaction products containing intact $\mathrm{B}$ chains do not mix with the A phase, the copolymers more or less penetrate only the $\mathrm{B}$ phase depending on the degree of polymerization of the bound A fragments. The concentration front for products on the left hand side therefore is nearly identical to the profile for non-reacting $\mathrm{B}(\mathrm{C})$ monomers. While, of course, the profile for the non-reacted $\mathrm{C}$ monomers of the simulation without reaction remains unchanged, the profiles of the reaction simulations penetrate the B phase, indicating the progress of consumption of reactive monomers. Since the reaction Split-End exhibits the greatest reaction rate in a large time section, the profile of the non-reacted $\mathrm{C}$ monomers is shifted more into the B phase compared to the other two reaction types. This is also the case for the concentration profiles of the reacted $\mathrm{C}$ monomers. For the same reasons the front of the reacted monomers for the reaction Split-End penetrates more into the B phase than those of the other two reaction types Split-Mid and Split-Ran. In every case, the profiles of the reacted $\mathrm{C}$ monomers label the position of reaction products of different type within the simulation box. Clearly, there is a indication for a double peak for all three reaction types, the first at $x \approx 3$, the second at $x \approx 15$, where definite reaction products must have been accumulated. We assume, that the first peak near the mathematical interface at $x=0$ can be associated to larger diblock copolymers, which are of restricted mobility only and remain near the position of their formation at the interface. We attribute the second peak to triblock copolymers exhibiting shorter A fragments which, therefore, are more miscible with the B phase and may diffuse deeper into the right part of the simulation box.

To illustrate this feature, for the reaction Split-End we have shown in Fig. (6) an analysis of the concentration profile of the reacted $\mathrm{C}$ monomers after termination of the simulation.

The behaviour of the two other interface reactions is similar to that of the reaction Split-End. As shown in the Figure, the essential contribution to the first peak near the interface originates from reacted $\mathrm{C}$ monomers in diblock copolymers, whereas the second peak widely stems from the corresponding $\mathrm{C}$ monomers in triblock copolymers. Note, that the concentration relation of reacted $\mathrm{C}$ monomers in diand triblock copolymers does not directly reflect the relation of the number of objects of the corresponding reaction products, since triblock copolymers contain two reacted C monomers. A detailed investigation of the formation behaviour of the reaction products shows, that the relation of the number of triblock copolymers to the number of diblock copolymers within the very first time section of the reaction is only $\approx 0.05$, but increases at the end of the simulation to a value of $\approx 0.6$. Diblock copolymers with relatively long $\mathrm{A}$ chains appear at the interface, whereas such molecules containing shorter A fragments also exist more to the right of the interface because of their greater miscibility with the B chains. The triblock copolymers generally exhibit a distinctly lower A content. Therefore the position of the centre of mass of the triblock copolymers is more shifted into the volume B phase. In the extreme case only two A monomers are located between the B chains. Note, however, there is an overlap of 


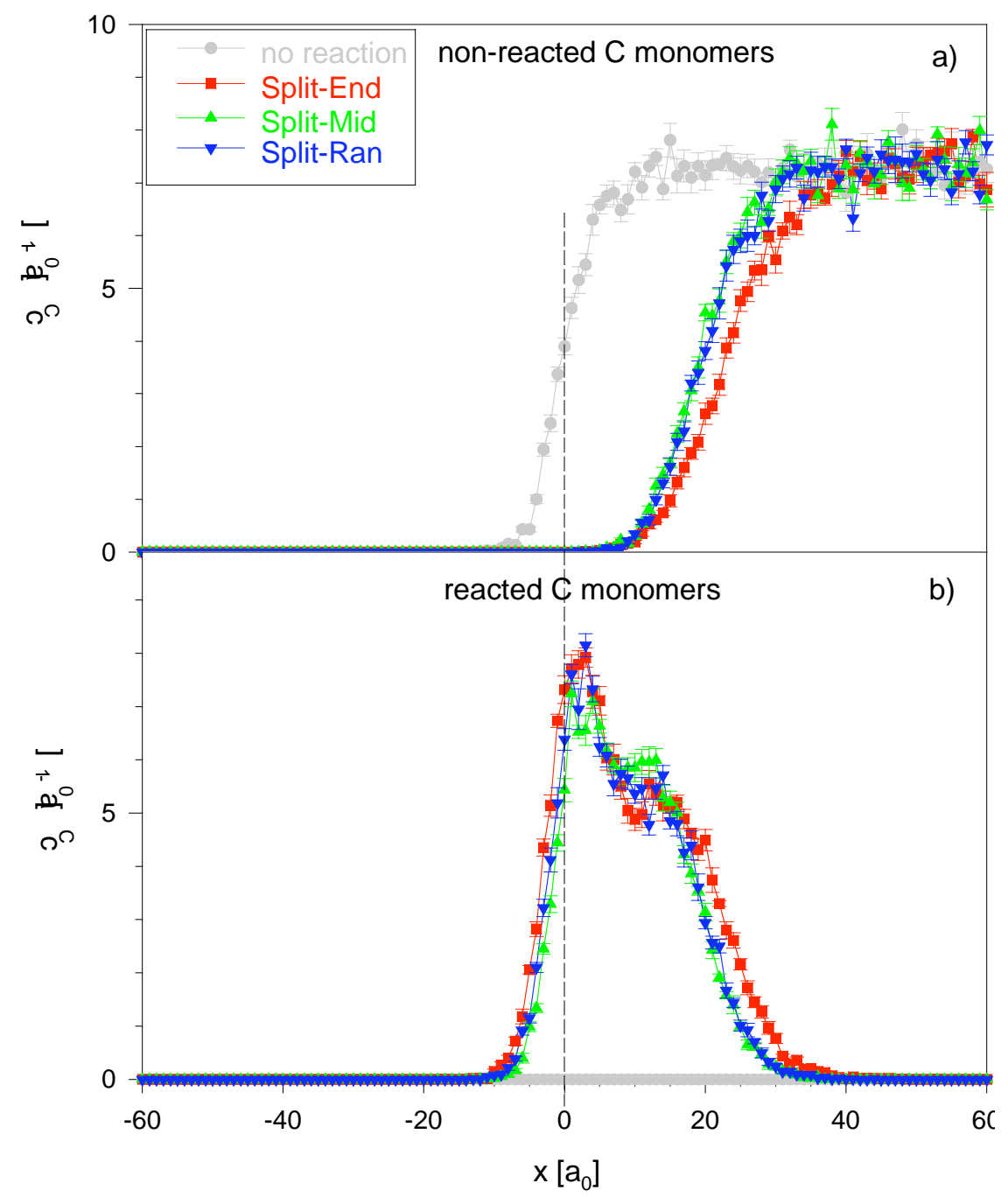

Fig. (5). Concentration profiles of the non-reacted (a) and reacted (b) C monomers for the reactions Split-End, Split-Mid and Split-Ran at activation energy $E_{A}=0 k_{B} T$ after termination of the simulation at time $=201813$ MCS compared to the system without reaction.

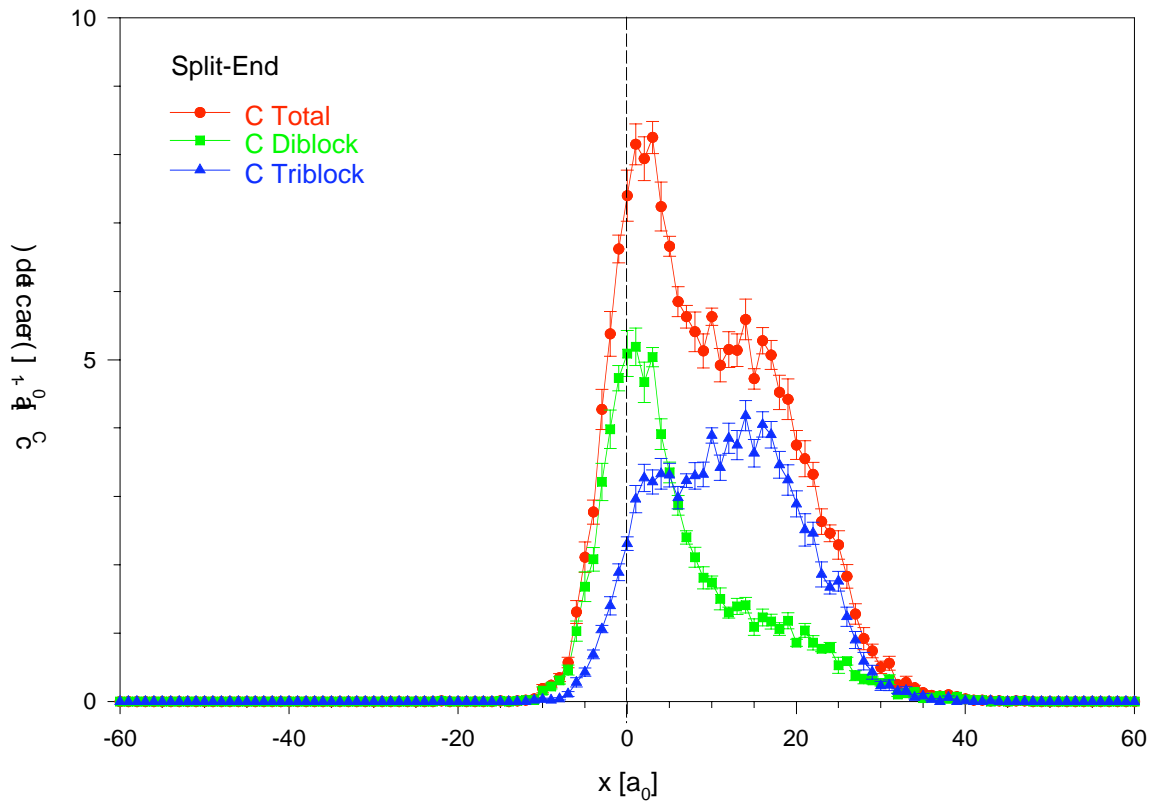

Fig. (6). The distribution of reacted C monomers (total, part of diblock copolymers, part of triblock copolymers) for the reaction Split-End after termination of the simulation at time $t=201813$ MCS. 
the local range of existence for the diblock and triblock copolymers to the right of the interface. Its width depends on the degree of polymerization of the A fragments in the block copolymers.

\section{ADHESION PROPERTIES}

In order to get some knowledge about adhesion properties of the composite on microscopic level within the framework of the Bond Fluctuation Model, we have adopted a bridge concept. In that concept tensile strength is associated with the number of connected monomers in $x$ direction and the number of block copolymers crossing the interface, respectively. To implement the bridge concept $f_{\text {simple }}$, for each monomer $M_{0}$ in every chain we count in $x$-direction the number of monomers $m_{L}$ to the left of the monomer $M_{0}$ and the number of monomers $m_{R}$ to the right of the monomer $M_{0}$ and form the product. In the concepts $f_{\text {unlike }}$ and $f_{\text {like }}$ we count additionally the number of contacts to monomers of different type in $r \leq \sqrt{6}$ in different chains $\left(f_{\text {unlike }}\right)$ und like chains $\left(f_{\text {like }}\right)$ providing a picture of the intra- and intermolecular monomer neighbourhood. For computational intentions this approach in detail reads:

$$
\begin{aligned}
& f_{\text {typ }}(x)=\sum_{i=1}^{N} f_{i}^{L}(x) \cdot f_{i}^{R}(x) \\
& f_{i}^{L}(x)=\sum_{j=1}^{P_{i}} \sum_{k=1}^{P_{i}} p_{i j k}(x) \quad f_{i}^{R}(x)=\sum_{j=1}^{P_{i}} \sum_{k=1}^{P_{i}} q_{i j k}(x) \\
& p_{i j k}(x)=\left\{\begin{array}{ll}
1+\Gamma_{t y p} & \text { for } x_{i k}<x_{i j} \\
0 & \text { for } x_{i k}=x_{i j}
\end{array}\right\} q_{i j k}(x)=\left\{\begin{array}{ll}
1+\Gamma_{t y p} & \text { for } x_{i k}>x_{i j} \\
0 & \text { for } x_{i k}=x_{i j}
\end{array}\right\}
\end{aligned}
$$

$\Gamma_{t y p}=\left\{\begin{array}{lll}0 & \text { for typ }=\text { simple } & \\ \rho & \text { for typ }=\text { unlike } & \text { number of unlike contacts in } r \leq \sqrt{6} \\ \sigma & \text { for typ }=\text { like } & \text { number of like contacts in } r \leq \sqrt{6}\end{array}\right\}$

with

unlike $=$ contacts of monomers $j, k$ to monomer $m$ of different type in chain $n \neq i$

like $=$ contacts of monomers $j, k$ to monomer $m$ of different type in chain $n=i$

and

$N$... Number of chains

$P_{i} \quad \ldots$ Degree of Polymerization of chain $i$

$p, q \quad \ldots$ Functions of the $x$ position of monomers $j, k$ in chain $i$

$\Gamma_{\text {typ }} \quad \ldots$ Weighting additive for contacts depending on

typ

$\rho, \sigma \quad \ldots$ Counter for weighting additive $\Gamma_{t y p}$

which implies, that the evaluation program is capable to identify the objects (single monomers, chains, branched chains etc.) to calculate the neighbourhood of the individual monomers.

We have compiled the contributions of all the three chemical reaction types for the three local resolved bridge concepts $f_{\text {simple }}, f_{\text {unlike }}$ and $f_{\text {like }}$ at three different simulation times in Figs. (7-9), respectively.

Since the bridge concepts are based on the principle of connectivity, the chemical reaction products with higher degree of polymerization than $P=32$ will extensively con-

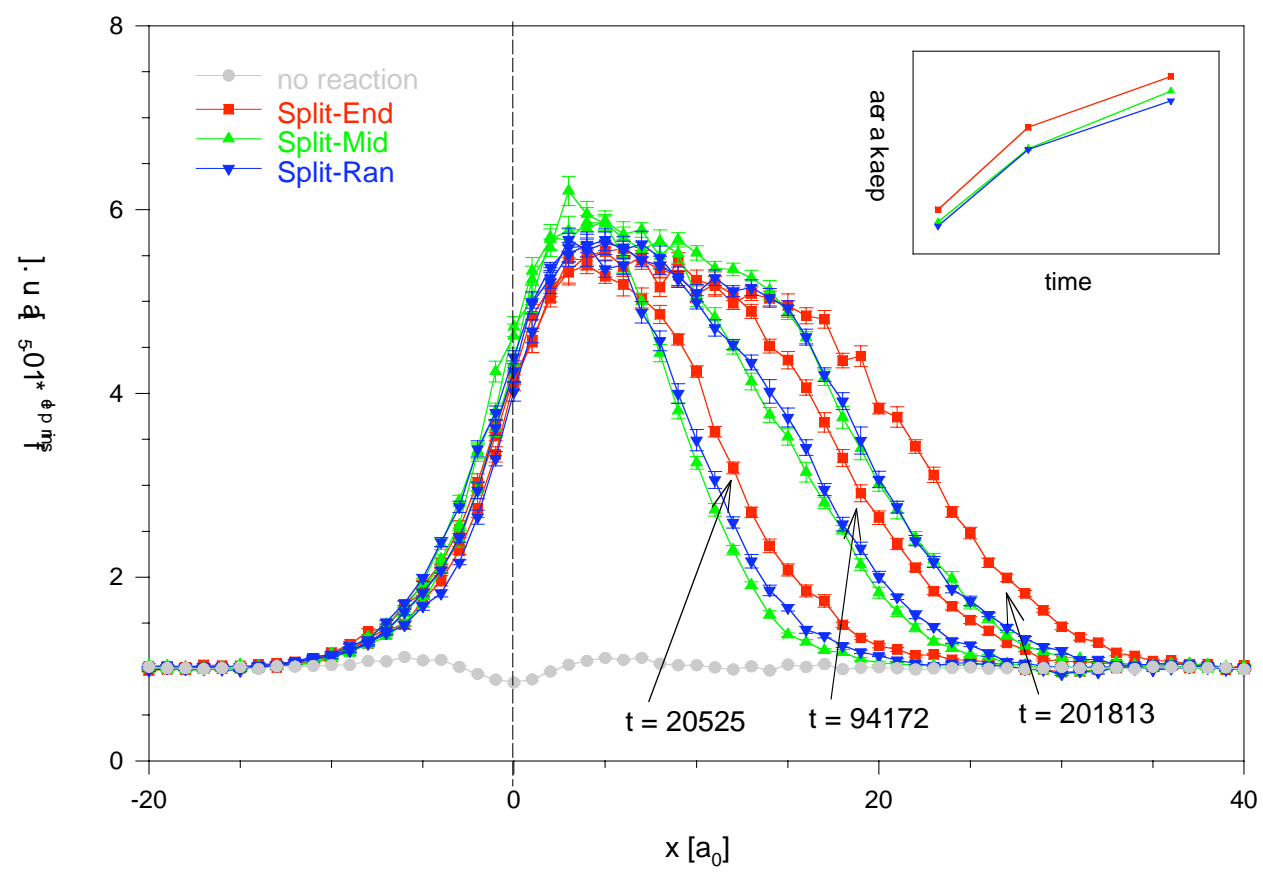

Fig. (7). The bridge concept $f_{\text {simple }}$ for the reactions Split-End, Split-Mid and Split-Ran at activation energy $E_{A}=0 \quad k_{B} T$ for the three time steps 20525, 94172 and 201813 MCS compared to the case without reaction. For the reactions, in the upper right corner the growth of the peak areas is shown schematically. 


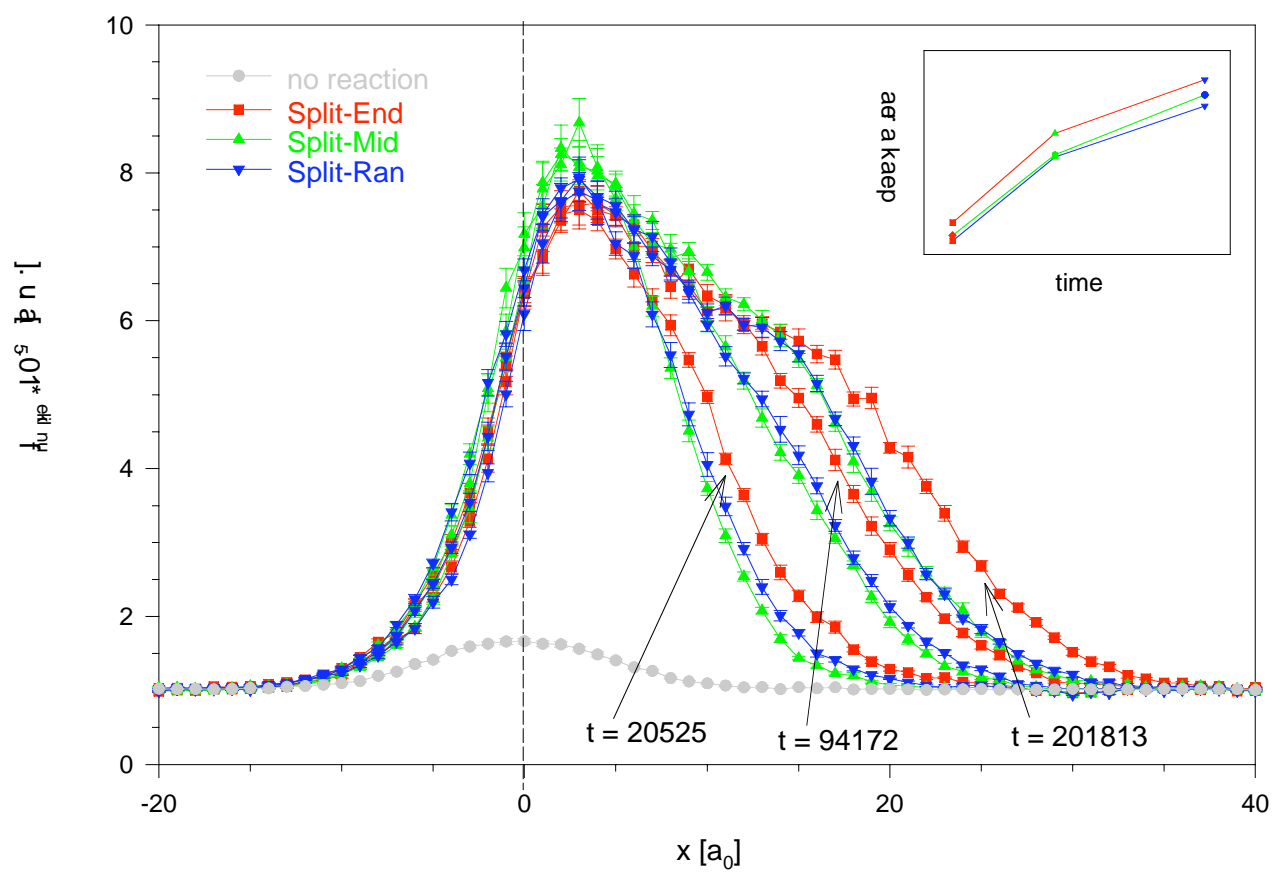

Fig. (8). The bridge concept $f_{\text {unlike }}$ for the reactions Split-End, Split-Mid and Split-Ran at activation energy $E_{A}=0 \quad k_{B} T$ for the three time steps 20525, 94172 and 201813 MCS compared to the case without reaction. For the reactions, in the upper right corner the growth of the peak areas is shown schematically.

tribute to the impression of strength enhancement, which surely will be overestimated by the large number of reactive sites in the molecules of type A. However, already the functions for the non-reactive system show some little effects in this context. So the function $f_{\text {unlike }}$ additionally weights the contacts of different monomers in different chains in the interphase region, which now is marked by a small maximum. Since the concepts $f_{\text {simple }}$ and $f_{\text {like }}$ in the case of the absence of reaction products yield the same results, the functions for these concepts are identical for the non-reactive system. The curves exhibit a flat minimum at $x=0$ and beside it two low maxima.

While the nature of the minimum at the interface may be found in a compression of the chains in $\mathrm{x}$ direction (which can be verified on calculating the dimension $\Delta x$ of the chains as a function of $\mathrm{x}$ ), the origin of the small maxima is determined by the enlarged concentration of chains on both sides of the interface in the non-reacted system (not shown here). As it was also observed for the concentration profiles of reaction products (reacted $\mathrm{C}$ monomers) and the distribution of the degree of polymerization, the peaks in the reacted systems appear to the right of the mathematical interface and, at least within the observation time, do not grow in height anymore, but into the B volume phase. While a small depression can be observed at the interface for the concept $f_{\text {simple }}$ without reaction (Fig. 7), the reactions strongly increase the strength to the right of the interdiffussion layer of the two phases. As indicated by the arrows, the growth of the function front occurs most rapidly for the reaction Split-End, followed by the other two functions for the reactions SplitMid and Split-Ran. This behaviour probably must be attributed to the higher average reaction rate of the reaction SplitEnd compared to the other two reactions due to the higher mobility of the reactive end-monomers.
The sketch in the upper right corner of the figure shows the increase of the peak areas with time as a measure for the total amount of bridging contacts. As expected, the reaction Split-End exhibits a higher number of bridges than the other two reactions Split-Mid and Split-Ran. A completely analogical behaviour can be observed for the bridge concepts $f_{\text {unlike }}$ and $f_{\text {like, }}$, where the contacts between $\mathrm{A}$ and $\mathrm{B}$ monomers in unlike $\left(f_{\text {unlike }}\right)$ and like $\left(f_{\text {like }}\right)$ molecules additionally highlight the interface region and in this way show the adhesive neighbourhood in real polymer systems. Note again, that the function values for both the concepts $f_{\text {unlike }}$ and $f_{\text {like }}$ within the volume phases approach the $f_{\text {simple }}$ limit, since the weight factors for $\mathrm{A} / \mathrm{B}$ contacts in the volume phases are always zero.

The main disadvantage of the three bridge concepts is the incapability to explain the relative strength between interface and volume part of the compound. According to these concepts the fracture should occur in the volume, but not near the interface, which in most cases is far away from practical observations. We believe to overcome this disproportion in later work by the development of more physically justified strength models. Possibly they can be based on the description of connecting forces between the phases, which rely on friction properties of the polymeric material.

The concept of bonding chains is not locally resolved in a direct way, but mediately counting only copolymers crossing the mathematical interface having one arm within the A phase and the other branch within the B phase: Let $x_{\min }$ and $x_{\max }$ label the minimum and maximum extension of the reaction product in $x$ direction, then the counter for the concept of bonding chains will increase by 1 when $x_{\min } \leq-R_{G}$ and $x_{\max } \geq+R_{G}$. Additionally, we divide the counter value by the total number of reaction events at the 


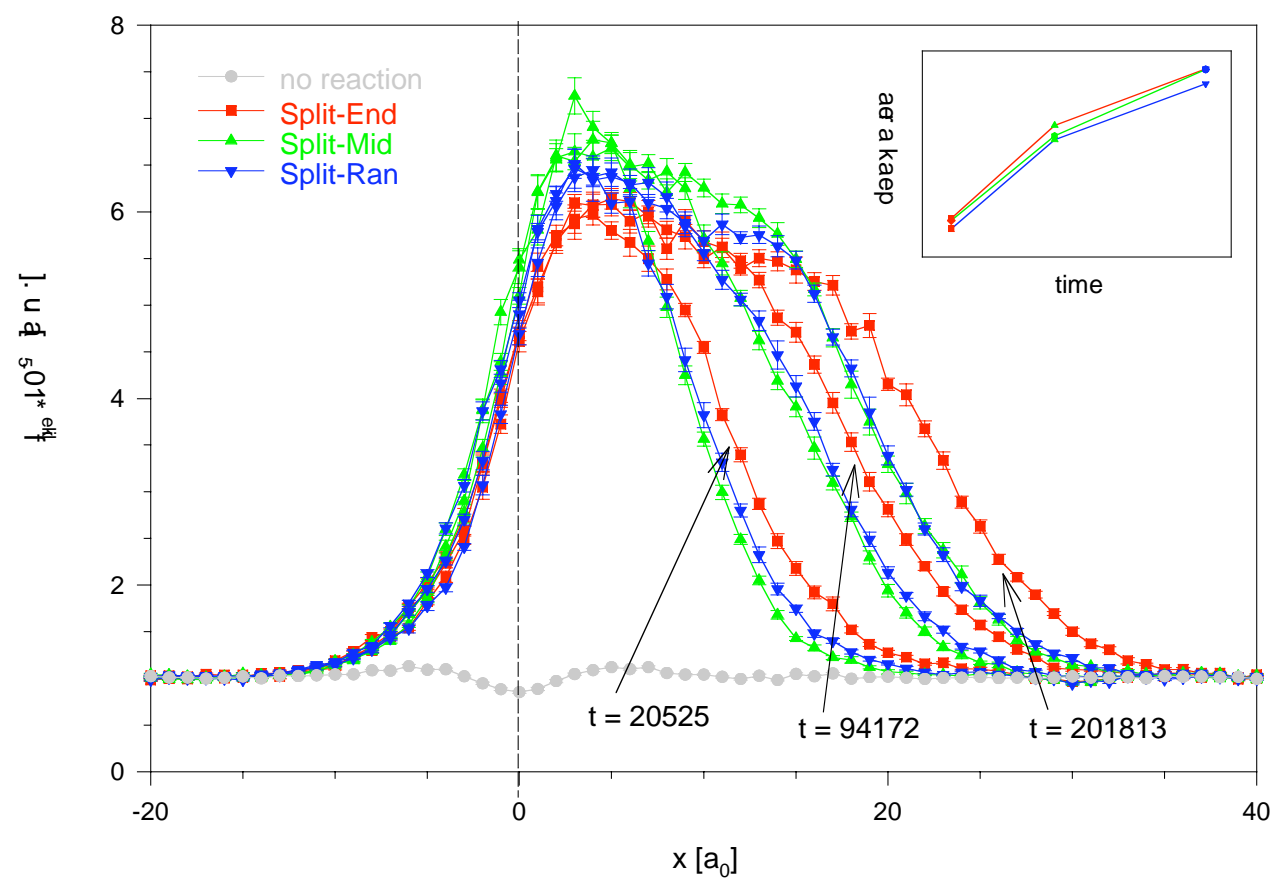

Fig. (9). The bridge concept $f_{\text {like }}$ for the reactions Split-End, Split-Mid and Split-Ran at activation energy $E_{A}=0 k_{B} T$ for the three time steps 20525, 94172 and 201813 MCS compared to the case without reaction. For the reactions, in the upper right corner the growth of the peak areas is shown schematically.

corresponding time, so that the function value is a measure for the efficiency of the reaction type in building such copolymers crossing the interface. This very illustrative concept with its high function values especially for short times, shows the benefit of the reactions Split-End and Split-Ran creating embedding reaction products in the very early state of the simulation. Chemical counterparts of these reaction types should therefore be used in the framework of the twocomponent injection molding.

\section{CONCLUSIONS}

In this Monte-Carlo simulation study based on the three dimensional coarse-grained bond fluctuation model we qualitatively characterized the behaviour of chemical reactions of the types (i) Split-End, (ii) Split-Mid and (iii) SplitRan being of importance for the reactive compatibilization in the two-component injection molding technique. All the irreversible reactions are governed by activation energies of $\mathrm{E}_{\mathrm{A}}=0,1,3,5$ and $7 k_{B} T$ successively slowing down the reaction speed. The reactions result in characteristic profiles for the degree of polymerization and the reaction products within the simulation volume, which are governed by the distribution of di- and triblock copolymers. Compiling the results for the bridge concepts $f_{\text {unlike }}, f_{\text {like }}, f_{\text {simple }}$, and the concept of the bonding chains, the reaction Split-End should lead to the most suitable behaviour reinforcing the interface of the two immiscible polymer melts and therefore should be the preferred reaction for compatibilization efforts in the two-component injection molding technique. For the future we intend to develop a more physically justified concept for the evaluation of the strength behaviour, than the simple bridge functions are able to establish.

\section{ACKNOWLEDGEMENTS}

Financial support from Deutsche Forschungsgemeinschaft (DFG) is acknowledged. We thank Dr. Michael Lang from the research group Theory of Polymers of the Leibniz Institut für Polymerforschung Dresden e. V. for helpful discussions.

\section{REFERENCES}

[1] Utracki, L., Polymer Alloys and Blends, Carl-Hanser-Verlag: New York, 1989.

[2] Wool, R.P., Polymer Interfaces: Structure and Strength, Carl Hanser Verlag: New York, 1995.

[3] Macosko, C.W.; Jeon, H.K.; Hoye, T.R. Reactions at polymerpolymer interfaces for blend compatibilization. Prog. Polym. Sci., 2005, 30(8-9), 939-947.

[4] Bates, F.S.; Fredrickson, G.H.. Block Copolymer Thermodynamics - Theory and Experiment. Annu. Rev. Phys. Chem., 1990, 41, 525557.

[5] Binder, K. Phase-Transitions in Polymer Blends and BlockCopolymer Melts - Some Recent Developments. Adv. Polym. Sci., 1994, 112, 181-299.

[6] Anastasiadis, S.H.; Gancarz, I.; Koberstein, J.T. Compatibilizing Effect of Block Copolymers Added to the Polymer-Polymer Interface. Macromolecules, 1989, 22(3), 1449-1453.

[7] Brown, S.B. Chemical processes applied to reactive extrusion of polymers. Anпи. Rev. Mater. Sci., 1991, 21, 409-435.

[8] Nishio, T.; Suzuki, Y.; Kojima, K.; Kakugo, M.J. Morphology of maleic-anhydride grafted polypropylene and polyamide alloy produced by reactive processing. Polym. Eng., 1991, 10(1-3), 123-149.

[9] Guegan, P.; Macosko, C.W.; Ishizone, T.; Hirao, A.; Nakahama, S. Kinetics of chain coupling at melt interfaces. Macromolecules, 1994, 27(18), 4993-4997.

[10] Nagel, J.; Scheidler, D.; Hupfer, B.; Bräuer, M.; Pleul, D.; Vogel, C.; Lehmann, D.; Amesöder, S. Investigations on the formation of composites by injection molding of PA6 and different grafted polypropylenes and their blends. J. Appl. Polym. Sci., 2006, 100(4), 2992-2999.

[11] Yin, Z.; Koulic, C.; Pagnoulle, C.; Jerome, R. Probing of the reaction progress at a PMMA/PS interface by using anthracene-labeled reactive PS chains. Langmuir 2003, 19(2), 453-457. 
[12] Jeon, H.K.; Macosko, C.W.; Moon, B.; Hoye, T.R.; Yin Z.; Coupling reactions of end- vs mid functional polymers. Macromolecules, 2004, 37(7), 2563-2571.

[13] Jones, T.D.; Schulze, J.S.; Macosko, C.W.; Lodge, T.P. Effect of thermodynamic interactions on reactions at polymer/polymer interfaces. Macromolecules, 2003, 36(19), 7212-7219.

[14] Nagel, J.; Bräuer, M.; Hupfer, B.; Grundke, K.; Schwarz, S.; Lehmann, D. Investigations on the reactive surface modification of polycarbonate by surface-reactive injection molding. J. Appl. Polym. Sci., 2004, 93(3), 1186-1191.

[15] Fredrickson, G.H. Diffusion-controlled reactions at polymerpolymer interfaces. Phys. Rev. Lett., 1996, 76(18), 3440-3443.

[16] O' Shaughnessy, B.; Sawhney, U. Polymer reaction kinetics at interfaces. Phys. Rev. Lett. 1996, 76(18), 3444-3447.

[17] Fredrickson, G.H.; Milner, S.T. Time-dependent reactive coupling at polymer-polymer interfaces. Macromolecules, 1996, 29(23), 7386-7390.

[18] O' Shaughnessy, B.; Vavylonis, D. Reactions at polymer interfaces: transitions from chemical to diffusion-control and mixed order kinetics. Europhys. Lett., 1999, 45(5), 638-644.

[19] Müller, M. Reactions at polymer interfaces: a monte-carlo simulation. Macromolecules, 1997, 30(20), 6353-6357.

[20] Yeung, C.; Herrmann, K.A. Molecular dynamics simulation of reactive compatibilization of polymer blends. Macromolecules, 2003, 36(1), 229-237.

[21] He, X.; Nagel, J.; Lehmann, D.; Heinrich, G. Interface structure between immiscible reactive polymers under transreaction: a monte-carlo simulation. Macromol. Theory Simul., 2005, 14(5), 305-311.

[22] Carmesin, I.; Kremer, K. The bond fluctuation method - a new effective algorithm for the dynamics of polymers in all spatial dimensions. Macromolecules, 1988, 21(9), 2819-2823.

[23] Deutsch, H.P.; Binder, K. Interdiffusion and self-diffusion in polymer mixtures - a monte-carlo study. J. Chem. Phys., 1991, 94(3), 2294-2304.

[24] Deutsch, H.P.; Binder, K. Critical-behavior and crossover scaling in symmetrical polymer mixtures - a monte-carlo investigation. Macromolecules, 1992, 25(23), 6214-6230.

[25] Müller, M. Miscibility behavior and single chain properties in polymer blends: a bond fluctuation model study. Macromol. Theory Simul., 1999, 8(4), 343-374.

[26] John, A.; Nagel, J.; Heinrich, G. Monte-carlo simulation of polymer reactions at interfaces. Macromol. Theory Simul., 2007, 16(4), 430-440.

[27] John, A.; Sommer, J.U. Monte-carlo simulation of the reactive formation of co-continuous nanostructured polymers. Macromol. Theory Simul., 2008, 17(6), 274-279.

[28] Metropolis, N.; Rosenbluth, A.N.; Rosenbluth, M.N.; Teller, A.H.; Teller, E. Equation of state calculations by fast computing machines. J. Chem. Phys., 1953, 21(6), 1087-1092.

[29] Kreer, T.; Baschnagel, J.; Müller, M.; Binder, K. Monte-carlo simulation of long chain polymer melts: crossover from rouse to reptation dynamics. Macromolecules, 2001, 34(4), 1105-1117.

(C) John et al.; Licensee Bentham Open.

This is an open access article licensed under the terms of the Creative Commons Attribution Non-Commercial License (http://creativecommons.org/licenses/by-nc/3.0/) which permits unrestricted, non-commercial use, distribution and reproduction in any medium, provided the work is properly cited. 